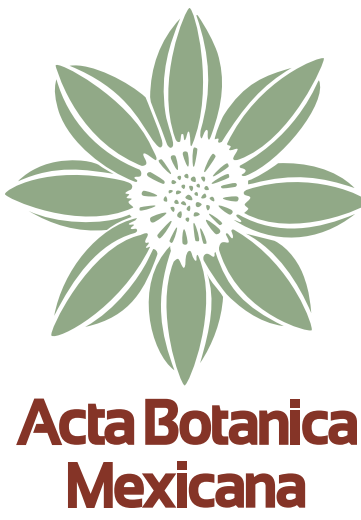

\title{
Un nuevo registro de Podagrostis (Poaceae, Pooideae, Poeae) para México
}

\section{A new record of Podagrostis (Poaceae, Pooideae, Poeae)} for Mexico

\author{
José Luis Vigosa-Mercado1,2
}

\section{Resumen:}

Antecedentes y Objetivos: El género Podagrostis incluye 12 especies, incluidas anteriormente en Agrostis y hasta hace poco se tenía conocimiento de la existencia de tres especies en México. La revisión de ejemplares de herbario reveló la presencia de una cuarta especie, conocida previamente solo de Guatemala. El objetivo de este trabajo es describir e ilustrar el nuevo registro, y discutir la identidad del ejemplar examinado y las diferencias morfológicas de esta especie con las otras mexicanas.

Métodos: Se revisaron ejemplares de herbario de Agrostis y Podagrostis de México y Centroamérica, en colecciones físicas y digitales. Se tomaron fotografías de las estructuras reproductivas importantes para la identificación.

Resultados clave: Se registra aquí por primera vez a Podagrostis exserta para México, en el estado de Oaxaca. Se discuten la identidad del ejemplar examinado y las diferencias de esta especie con las otras especies mexicanas de Podagrostis. Se presenta una clave dicotómica para diferenciarlas.

Conclusiones: El género Podagrostis está representado en México por cuatro especies: P. exserta, P. liebmannii, P. novogaliciana y P. rosei, que se distinguen de Agrostis por las espiguillas con la raquilla prolongada. Se destaca la importancia de la labor curatorial en las colecciones de los herbarios mexicanos.

Palabras clave: florística, gramíneas, Oaxaca, taxonomía.

\section{Abstract:}

Background and Aims: The genus Podagrostis includes 12 species, formerly included in Agrostis, and until recently, only the existence of three species was known from Mexico. The examination of herbarium specimens revealed the presence of a fourth species, previously known only from Guatemala. The aim of this work is to describe and illustrate the new record, and to discuss the identity of the examined specimen and the morphological differences of this species with the other Mexican species.

Methods: Herbarium specimens of Agrostis and Podagrostis of Mexico and Central America, from physical and digital collections, were examined and photographs of important reproductive structures for identification were taken.

Key results: Podagrostis exserta is recorded here for the first time in Mexico, in the state of Oaxaca. The identity of the examined specimen and the differences of this species with other Mexican species of Podagrostis are discussed. A dichotomous key is presented to differentiate them.

Conclusions: The genus Podagrostis is now represented in Mexico by four species, P. exserta, P. liebmannii, P. novogaliciana and $P$. rosei, distinguishable from Agrostis by spikelets with a prolonged rachilla. The importance of curatorial work of Mexican herbaria collections is highlighted.

Key words: floristics, grasses, Oaxaca, taxonomy.

${ }^{1}$ Universidad Nacional Autónoma de México, Instituto de Biología, Posgrado en Ciencias Biológicas, Ciudad Universitaria, Av. Universidad 3000, Coyoacán, 04510 Cd. Mx., México.

${ }^{2}$ Autor para la correspondencia: luis_vigosa@ciencias. unam.mx
Recibido: 26 de octubre de 2021

Revisado: 11 de noviembre de 2021.

Aceptado por Marie-Stéphanie Samain: 8 de diciembre de 2021.

Publicado Primero en línea: 17 de diciembre de 2021. Publicado: Acta Botanica Mexicana 129 (2022).
Citar como: Vigosa-Mercado, J. L. 2021(2022). Un nue vo registro de Podagrostis (Poaceae, Pooideae, Poeae) para México. Acta Botanica Mexicana 129: e1993. DOI: https://doi.org/10.21829/abm129.2022.1993 


\section{Introducción}

El género Podagrostis (Griseb.) Scribn. \& Merr. incluye 12 especies, distribuidas desde las costas de Alaska hasta Chile y Argentina (Molina et al., 2021). Sus especies fueron consideradas como parte de Agrostis L. (p. ej., Clayton y Renvoize, 1986; Pohl y Davidse, 1994) y recientemente evidencia morfológica y molecular ha permitido su reconocimiento como un género distinto (Peterson et al., 2020; Sylvester et al., 2020; Molina et al., 2021). Podagrostis se identifica por la siguiente combinación de características: espiguillas unifloras con el flósculo tan largo o ligeramente más corto que las glumas, pálea bien desarrollada y raquiIla prolongada a manera de cerda glabra o pilosa distalmente. En cuanto a su clasificación, el género se ubica en la familia Poaceae, subfamilia Pooideae, tribu Poeae y subtribu Agrostidinae (Soreng et al., 2017).

En México se ha registrado la presencia de tres especies, P. novogaliciana (McVaugh) A. M. Molina \& Rúgolo, P. liebmannii (E. Fourn.) Sylvester \& Soreng y P. rosei (Scribn. \& Merr.) Sylvester \& Soreng (Molina et al., 2021), todas registradas como Agrostis en revisiones recientes de la familia en el país (Dávila et al., 2018; Sánchez-Ken, 2018(2019)). La primera especie se conoce del estado de Jalisco, la segunda de Hidalgo, Puebla y Veracruz, mientras que la tercera de Durango y Zacatecas (Fig. 1).

Durante la revisión de ejemplares de herbario de Agrostis, se detectó la presencia de una cuarta especie en México: P. exserta (Swallen) Sylvester \& Soreng, conocida previamente solo de Guatemala. El objetivo de este trabajo es describir e ilustrar el nuevo registro, y discutir la identidad del ejemplar examinado y las diferencias morfológicas de esta especie con otras mexicanas.

\section{Materiales y Métodos}

Se revisaron ejemplares de herbario de Agrostis y Podagrostis de México y Centroamérica, de manera presencial, depositados en las siguientes colecciones físicas: CHAPA (Colegio de Posgraduados), ENCB (Escuela Nacional de Ciencias Biológicas), FCME (Facultad de Ciencias de la Universidad Nacional Autónoma de México), IEB (Instituto de Ecología, A.C., Centro Regional del Bajío) y MEXU (Herbario Nacional de México) (abreviaturas según Thiers, 2021). También se revisaron ejemplares en las siguientes colec- ciones digitales: New York Botanical Garden (NY, 2021), Museo Nacional de Historia Natural, de Francia (P, 2021) y Herbario Nacional de los Estados Unidos de América (US, 2021). Se midieron estructuras para la elaboración de una descripción morfológica, la cual también fue complementada con literatura (Pohl y Davidse, 1994; Sylvester et al., 2020; Molina et al., 2021). Finalmente, se tomaron fotografías de las estructuras reproductivas en un microscopio estereoscópico (Velab VE-S1, Cd. Mx., México), con un teléfono celular (iPhone @Apple, SE 2 a generación, California, EUA).

\section{Resultados}

\section{Taxonomía}

Podagrostis exserta (Swallen) Sylvester \& Soreng, PhytoKeys 148: 34. 2020. Figs. 2, 3.

$\equiv$ Agrostis exserta Swallen, Contr. U.S. Natl. Herb. 29(9): 404. 1950. TIPO: GUATEMALA. Huehuetenango, collected in alpine area, vicinity of Tojquia, Sierra de los Cuchumatanes, 3700 m, 5.VIII.1942, J. A. Steyermark 50119 (holotipo: US00131747, isotipo: US00131748).

Hierbas cespitosas, hasta $30 \mathrm{~cm}$ de alto; culmos erectos, simples, glabros, sin nudos expuestos, renuevos intravaginales; hojas basales, vainas con márgenes libres, glabras, las inferiores fibrosas con la edad, lígulas 0.8-2(2.5) $\mathrm{mm}$ de largo, membranáceas, ápice agudo a truncado, erosas, láminas 1-7.5 cm de largo, 0.3-0.6 mm de ancho, filiformes, involutas, escabrosas abaxialmente; sinflorescencias en forma de panículas (1.2-)2-5(-5.7) cm de largo, (0.51-2.5 cm de ancho, ligeramente contraídas a extendidas en la madurez, ramas ascendentes a divergentes, glabras, las más largas hasta $2 \mathrm{~cm}$ de largo, pedicelos $1-5 \mathrm{~mm}$ de largo, glabros, ascendentes a divergentes; espiguillas (1.2)1.5-2 mm de largo, con 1 flósculo; glumas (1.2-)1.5-2 mm de largo, subiguales, lanceoladas, ligeramente aquilladas, ápice agudo, escabriúsculas en la quilla hacia el ápice, con 1(3) nervaduras; lema (1.1-)1.4-1.7 mm de largo, tan larga o ligeramente más corta que las glumas, elíptica, ápice obtuso, glabra, con 5 nervaduras, sin arista o con 1 arista dorsal, hasta $0.5 \mathrm{~mm}$ de largo, inserta hacia la mitad o en 


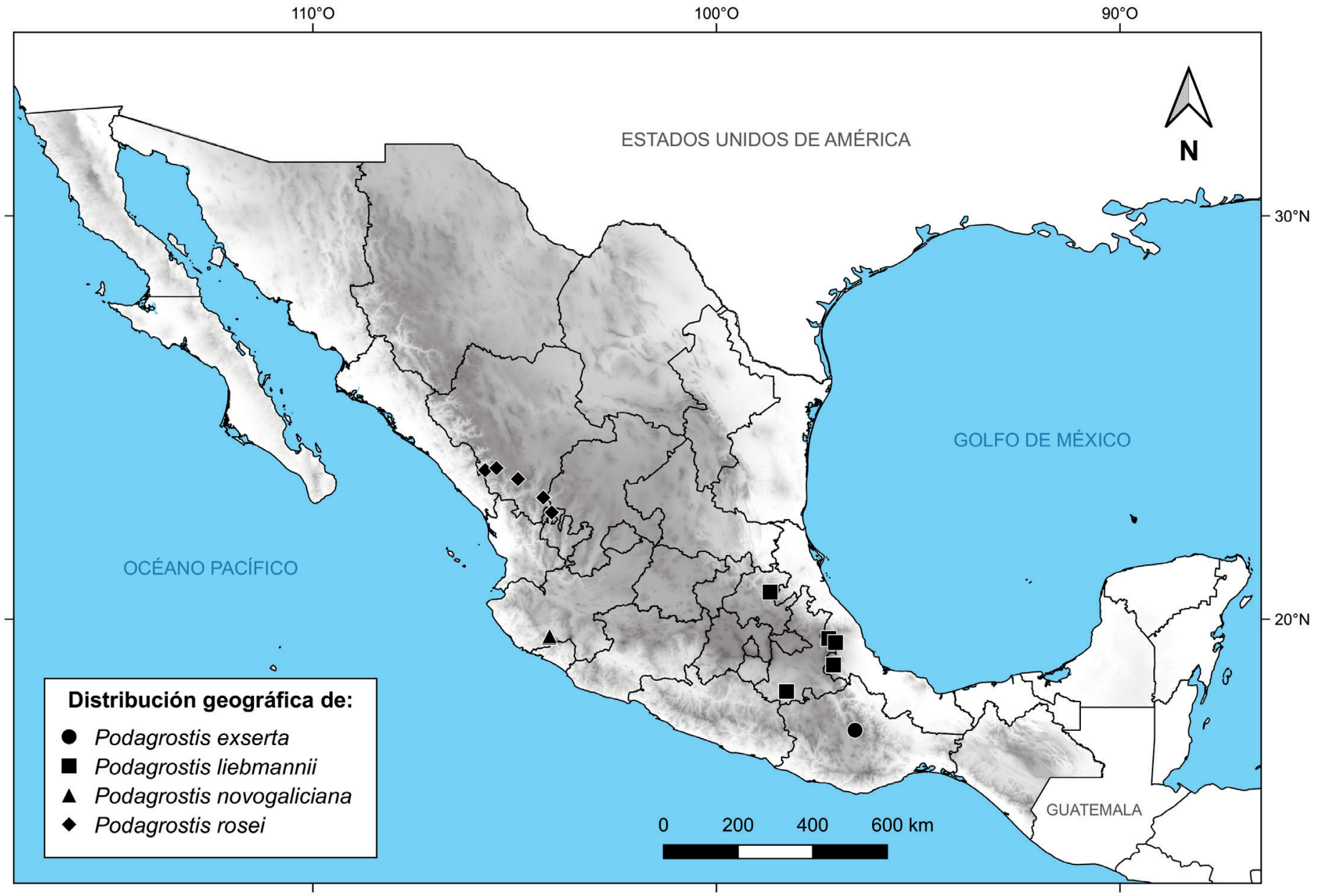

Figura 1: Distribución geográfica conocida del género Podagrostis (Griseb.) Scribn. \& Merr. en México.

el tercio superior; callo presente, glabro; pálea 0.9-1.3 mm de largo, oblonga, ligeramente biaquillada, ápice eroso, glabra, sin nervaduras; raquilla prolongada en la base del flósculo, 0.3-0.5 mm, lisa o escabrosa; anteras 3, 0.7-1.1 $\mathrm{mm}$ de largo; frutos no observados.

Distribución y hábitat: planta conocida de Guatemala y México (Oaxaca) (Fig. 1). En México se ha colectado en sitios abiertos en bosque de coníferas, a una altitud de ca. $2300 \mathrm{~m}$.

Material examinado: GUATEMALA. Huehuetenango, Sierra de los Cuchumatanes, between Tojiah and Chemal at km 319.5 on Ruta Nacional 9N, 3380 m, in grassy meadow, J. H. Beaman 3873 (US-2381699); Todos Santos, cerca de La Torre, 3700 m, bosque de pino, J. Gálvez et al. 96.5813
(MEXU-869047); San Juan Ixcoy, Chanchocal, 3200 m, pradera, 11.X.2000, M. Véliz et al. 2M.10152 (MEXU-992471, MEXU-1051385). MÉXICO. Oaxaca, 27 km de Oaxaca rumbo a Tuxtepec, bosque aciculi-linearifolio (bosque mixto de coníferas), 1.VII.1980, A. A. Beetle M-5004 (MEXU1088668).

\section{Discusión}

Podagrostis exserta no había sido registrada anteriormente en México bajo su nombre aceptado, ni el de sus sinónimos, ni en catálogos recientes de la familia Poaceae a nivel nacional (Dávila et al., 2018; Sánchez-Ken, 2018(2019)), ni en trabajos a nivel estatal (Dávila et al., 1990; EspinosaGarduño y Rodríguez-Jiménez, 1996; Herrera-Arrieta y Rzedowski, 2001; Herrera Arrieta et al., 2010; Zamudio y Galván-Villanueva, 2011; Pacheco et al., 2012; Herrera- 


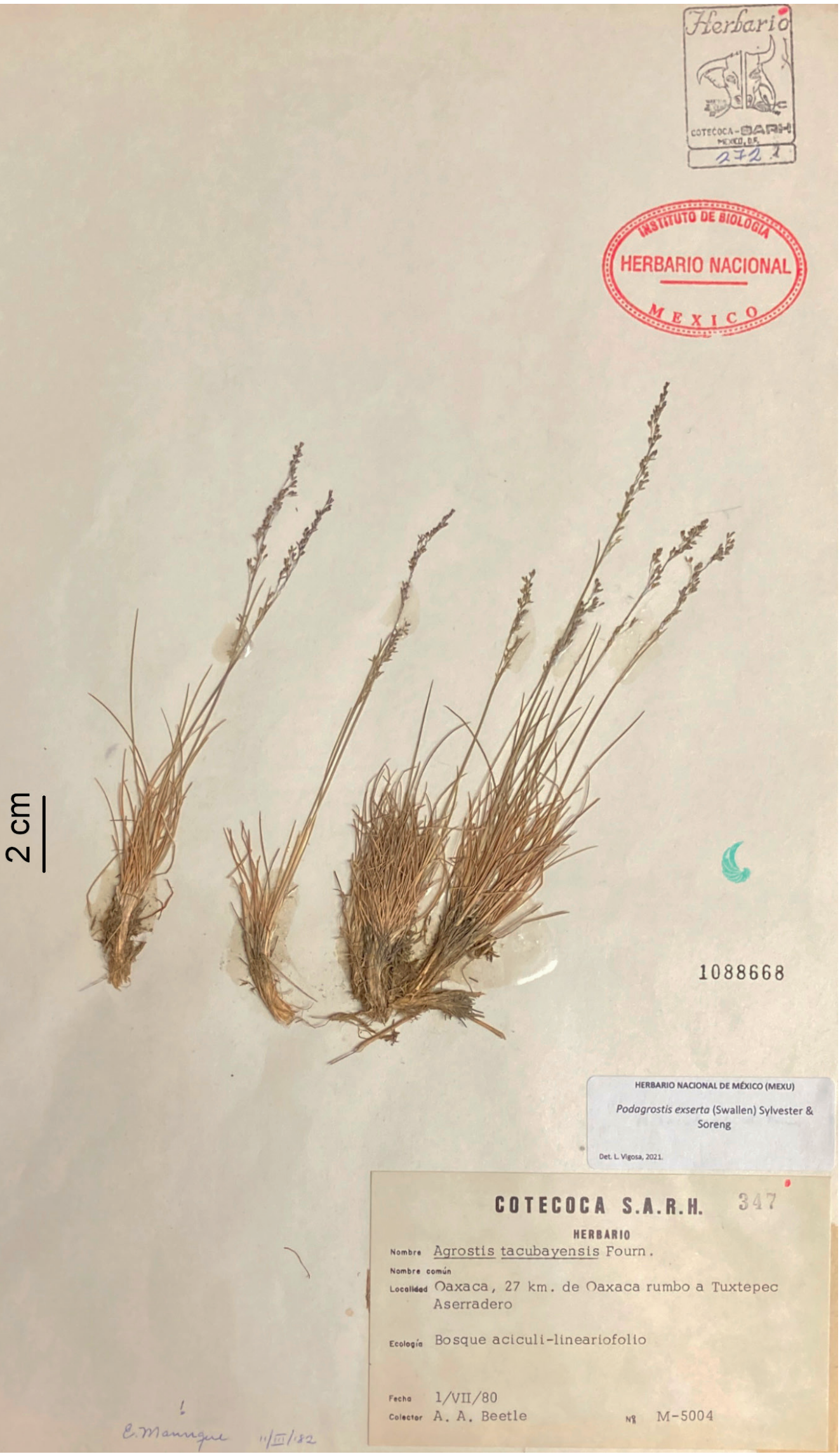

Figura 2: Podagrostis exserta (Swallen) Sylvester \& Soreng. Beetle M-5004 (MEXU). 

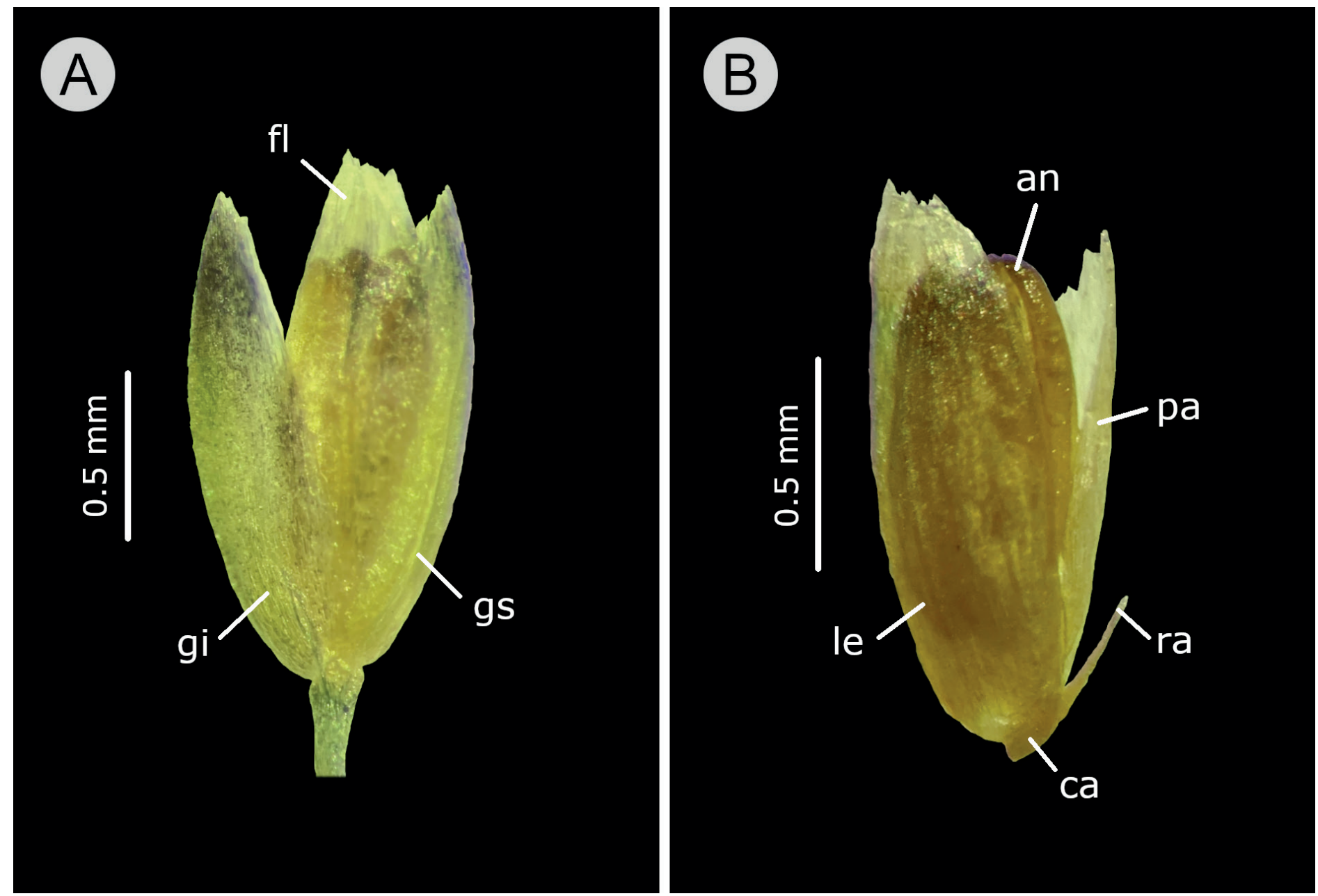

Figura 3: Podagrostis exserta (Swallen) Sylvester \& Soreng. A. espiguilla; B. flósculo. an=anteras, ca=callo, fl=flósculo, gi=gluma inferior, gs=gluma superior, le=lema, pa=pálea, ra=raquilla. Composición basada en Beetle M-5004 (MEXU).

Arrieta, 2014; Valdés-Reyna, 2015; Sánchez-Ken y CerrosTlatilpa, 2016; Herrera Arrieta y Peterson, 2018; Rzedowski y Bedolla-García, 2021).

Los registros previos de la especie indicaban que solamente se encontraba en las regiones montañosas de Guatemala (Pohl y Davidse, 1994; Sylvester et al., 2020). El ejemplar examinado (Beetle M-5004), colectado en el estado de Oaxaca, se encontraba identificado erróneamente como Agrostis tacubayensis E. Fourn. Además, fue citado e ilustrado bajo dicho nombre en el volumen I de la obra Las Gramíneas de México (Beetle, 1983). Actualmente este nombre se considera como un sinónimo de $A$. schaffneri E. Fourn. Las espiguillas del ejemplar examinado cuentan con una pálea bien desarrollada y la raquilla prolongada a manera de cerda glabra. Ninguna de estas característi- cas se encuentra en $A$. schaffneri. Otras características que permiten ubicar al ejemplar en Podagrostis exserta son las hojas basales, con láminas filiformes. Por otro lado, las sinflorescencias del ejemplar examinado son angostas, mientras que típicamente en $P$. exserta son ovoides y abiertas. Esta diferencia puede deberse a que en el ejemplar las sinflorescencias son inmaduras. Las demás características coinciden con lo registrado previamente para la especie (Pohl y Davidse, 1994; Sylvester et al., 2020).

Podagrostis exserta se distingue de las otras especies mexicanas por sus hojas basales, con las láminas filiformes e involutas y las espiguillas de menor tamaño. Se proporciona una clave detallada para distinguir las especies mexicanas del género Podagrostis. 
Clave para distinguir las especies mexicanas de Podagrostis (modificada de Sylvester et al., 2020)

1a. Hojas basales, láminas todas filiformes, involutas; espiguillas (1.2-)1.5-2 mm de largo; plantas de México (Oaxaca) y Guatemala

P. exserta (Swallen) Sylvester \& Soreng

1b. Hojas basales y/o caulinares, láminas de los culmos lineares y planas, las de los renuevos en ocasiones filiformes e involutas; espiguillas 1.8-3.5 mm de largo; plantas de México (Durango, Hidalgo, Jalisco, Puebla, Veracruz y Zacatecas) .. 2

2a. Plantas largamente rizomatosas; espiguillas $3-3.5 \mathrm{~mm}$ de largo; glumas lisas en las quillas; lemas ligeramente coriáceas en la madurez; plantas de Jalisco

P. novogaliciana (McVaugh) A. M. Molina \& Rúgolo

2b. Plantas cespitosas; espiguillas 1.8-2.3 mm de largo; glumas escabriúsculas en las quillas; lemas membranáceas en la madurez; plantas de Durango, Hidalgo, Puebla, Veracruz y Zacatecas 3

3a. Lemas con una arista dorsal, 1.6-2 mm largo, inserta en el tercio inferior, recta o geniculada; plantas de Durango y Zacatecas

P. rosei (Scribn. \& Merr.) Sylvester \& Soreng

3b. Lemas sin arista; plantas de Hidalgo, Puebla y Veracruz P. liebmannii (E. Fourn.) Sylvester \& Soreng

\section{Conclusiones}

El género Podagrostis está representado en México por cuatro especies: $P$. exserta, $P$. liebmannii, $P$. novogaliciana y $P$. rosei. Se distinguen de Agrostis por las espiguillas con la raquilla prolongada. El nuevo registro se conoce solamente de un ejemplar de herbario colectado en el estado de Oaxaca. No se ha vuelto a colectar recientemente. No se cuenta con suficiente información para proponer una categoría de riesgo. A manera de conclusión, se destaca la importancia de la labor curatorial de los especialistas en las colecciones de los herbarios mexicanos, debido a que el nuevo registro se basa en una colecta que no había sido identificada correctamente, a pesar de que fue colectada hace más de 30 años.

\section{Contribución de autores}

JLVM concibió, diseñó y realizó el estudio, escribió y revisó el manuscrito.

\section{Financiamiento}

El autor cuenta con el apoyo de una beca del Consejo Nacional de Ciencia y Teconología (CONACYT, CVU 778698).

\section{Agradecimientos}

El autor agradece a los encargados de los herbarios CHAPA, ENCB, FCME, IEB y MEXU, por las facilidades otorgadas para la consulta de sus colecciones, particularmente a Gilda Ortiz Calderón. A Steven Ramírez por la revisión del resumen en inglés. A Abisaí García Mendoza y un revisor anónimo por los comentarios que permitieron mejorar la primera versión del manuscrito.

\section{Literatura citada}

Beetle, A. A., R. Guzmán, V. Jaramillo, M. P. Guerrero, E. Manrique, A. Chimal, C. Shariff e I. Núñez. 1983. Las Gramíneas de México I. Secretaría de Agricultura y Recursos Hidraúlicos y Comisión Técnico Consultiva para la Determinación de Coeficientes de Agostadero. Cd. Mx., México. 260 pp.

Clayton, W. D. y S. A. Renvoize. 1986. Genera Graminum, grasses of the world. Kew Bulletin, Additional Series 13: 1-389.

Dávila, P., M. T. Mejía-Saulés, A. M. Soriano-Martínez y Y. HerreraArrieta. 2018. Conocimiento taxonómico de la familia Poaceae en México. Botanical Sciences 96(3): 462-514. DOI: https://doi.org/10.17129/botsci.1894

Dávila, P., P. Tenorio, E. Manrique, A. Miranda y A. Rodríguez. 1990. Las gramíneas de Puebla. Instituto de Biología, Universidad Nacional Autónoma de México. Cd. Mx., México. 51 pp.

Espinosa-Garduño, J. y L. S. Rodríguez-Jiménez. 1996. Listado florístico del estado de Michoacán sección IV (Angiospermae: Fagaceae, Gramineae, Krameriaceae, Leguminosae). Flora del Bajío y de Regiones Adyacentes XII: 1-271.

Herrera-Arrieta, Y. 2014. Additions and updated names for grasses of Durango, Mexico. Acta Botanica Mexicana 106: 79-95. DOI: https://doi.org/10.21829/abm106.2014.214

Herrera Arrieta, Y. y P. M. Peterson. 2018. Grasses of Chihuahua, Mexico. Smithsonian Contributions to Botany 107: 1-308. DOI: https://doi.org/10.5479/si.1938-2812.107

Herrera-Arrieta, Y. y J. Rzedowski. 2001. Poaceae: In: Calderón de Rzedowski, C. y J. Rzedowski (eds.). Flora fanerogámica del Valle de México. Instituto de Ecología, A.C. y Comisión Nacional para el Conocimiento y Uso de la Biodiversidad. Cd. Mx., México. Pp. 999-1114. 
Herrera Arrieta, Y., P. M. Peterson y A. Cortés Ortiz. 2010. Gramíneas de Zacatecas, México. Sida, Botanical Miscellany 32: 1-239.

Molina, A. M., Z. E. Rúgolo, M. A. Ferrero, P. C. Palacio, C. Guerreiro y A. S. Vega. 2021. Taxonomic revision of the genus Podagrostis (Poaceae, Pooideae, Poeae). Annals of the Missouri Botanical Garden 106: 245-270. DOI: https:// doi.org/10.3417/2021642

NY. 2021. C. V. Starr Virtual Herbarium. New York Botanical Garden. New York, USA. http://sweetgum.nybg.org/ science/vh/ (consultado octubre de 2021).

P. 2021. Vascular Plants Collection. Muséum National d'Histoire Naturelle. Paris, France. https://science.mnhn.fr/ institution/mnhn/collection/p/ (consultado octubre de 2021).

Pacheco, D., P. Dávila y M. T. Mejía-Saulés. 2012. Poaceae. In: García-Mendoza, A. y J. Meave (eds.). Diversidad florística de Oaxaca: de Musgos a Angiospermas. Universidad Nacional Autónoma de México y Comisión Nacional para el Conocimiento y Uso de la Biodiversidad. Cd. Mx., México. Pp. 339-349.

Peterson, P. M., S. P. Sylvester, K. Romaschenko, R. J. Soreng, P. Barberá, A. Quintanar y C. Aedo. 2020. A phylogeny of species near Agrostis supporting the recognition of two new genera, Agrostula and Alpagrostis (Poaceae, Pooideae, Agrostidinae) from Europe. Phytokeys 167: 57-82. DOI: https://doi.org/10.3897/phytokeys.167.55171

Pohl, R. W. y G. Davidse. 1994. Agrostis. In: Davidse, G., M. SousaSánchez y A. O. Chater (eds.). Flora Mesoamericana, Vol. 6. Universidad Nacional Autónoma de México. Cd. Mx., México. Pp. 237-240.

Rzedowski, J. y B. Y. Bedolla-García. 2021. Catálogo de especies de plantas vasculares registradas del estado de Querétaro. Flora del Bajío y de Regiones Adyacentes XXXVII: 1-166.
Sánchez-Ken, J. G. 2018(2019). Riqueza de especies, clasificación y listado de las gramíneas (Poaceae) de México. Acta Botanica Mexicana 126: e1379. DOI: https://doi.org/10.21829/ abm126.2019.1379

Sánchez-Ken, J. G. y R. Cerros-Tlatilpa. 2016. Listado florístico de la familia Poaceae en el estado de Morelos, México. Acta Botanica Mexicana 116: 65-105. DOI: https://doi. org/10.21829/abm116.2016.1119

Soreng, R. J., P. M. Peterson, K. Romaschenko, G. Davidse, J. K. Teisher, L. G. Clark, P. Barberá, L. J. Gillespie y F. O. Zuloaga. 2017. A worldwide phylogenetic classification of the Poaceae (Gramineae) II: An update and a comparison of two 2015 classifications. Journal of Systematics and Evolution 55(4): 259-290. DOI: https://doi.org/10.1111/jse.12262

Sylvester, S. P., P. M. Peterson, K. Romaschenko, W. J. BravoPedraza, L. E. Cuta-Alarcón y R. J. Soreng. 2020. New combinations and updated descriptions in Podagrostis (Agrostidineae, Poaceae) from the Neotropics and Mexico. PhytoKeys 148: 21-50. DOI: https://doi.org/10.3897/ phytokeys.148.50042

Thiers, B. 2021. Index Herbariorum: A global directory of public herbaria and associated staff. New York Botanical Garden's Virtual Herbarium. New York, USA. http://sweetgum.nybg. org/science/ih/ (consultado octubre de 2021).

US. 2021. National Museum of Natural History. Botany Collections. Smithsonian Institution. Washington, D.C., USA. https:// collections.nmnh.si.edu/search/botany/ (consultado octubre 2021).

Valdés-Reyna, J. 2015. Gramíneas de Coahuila. Comisión Nacional para el Conocimiento y Uso de la Biodiversidad. Cd. Mx., México. 556 pp.

Zamudio, S. y R. Galván Villanueva. 2011. La diversidad vegetal del estado de Guanajuato, México. Flora del Bajío y de Regiones Adyacentes XXVII: 1-101. 